\title{
Pratiques
}

Linguistique, littérature, didactique

\section{Quand Dragon accouche le « déjà-là » du texte : l'apport d'un logiciel de reconnaissance vocale dans une activité de rédaction de résumé}

When Dragon delivers the "déjà-là" of the text: the contribution of a speech recognition software in an activity of summary

Marie-Emmanuelle Pereira, Christina Romain et Véronique Rey

\section{OpenEdition}

Journals

Édition électronique

URL : http://journals.openedition.org/pratiques/3410

DOI : $10.4000 /$ pratiques.3410

ISSN : 2425-2042

Éditeur

Centre de recherche sur les médiations (CREM)

Référence électronique

Marie-Emmanuelle Pereira, Christina Romain et Véronique Rey, « Quand Dragon accouche le « déjà-là » du texte : l'apport d'un logiciel de reconnaissance vocale dans une activité de rédaction de résumé », Pratiques [En ligne], 173-174 | 2017, mis en ligne le 10 mars 2017, consulté le 21 avril 2019. URL : http://journals.openedition.org/pratiques/3410; DOI : 10.4000/pratiques.3410

Ce document a été généré automatiquement le 21 avril 2019

(C) Tous droits réservés 


\title{
Quand Dragon accouche le " déjà-là » du texte : l'apport d'un logiciel de reconnaissance vocale dans une activité de rédaction de résumé
}

\author{
When Dragon delivers the "déjà-là" of the text: the contribution of a speech \\ recognition software in an activity of summary
}

Marie-Emmanuelle Pereira, Christina Romain et Véronique Rey

1 L'écriture et la parole sont traditionnellement présentées comme un couple dont chacun des membres est paré de vertus qui font défaut à l'autre. Le mythe de l'invention de l'écriture proposé par Platon dans le Phèdre donne à voir cet art comme une externalisation de la mémoire humaine, et aux discours écrits n'est accordée que l'apparence de la vie. Dans son Essai sur l'origine des langues, J.-J. Rousseau (1990, p. 79) regrettera quant à lui que l'écriture altère la langue en la fixant, et «substitue l'exactitude à l'expression ». Les questions d'externalisation de la mémoire humaine et d'exactitude, si elles sont l'objet d'une dévalorisation par Platon et J.-J. Rousseau, ne sont pas sans lien avec la raison graphique travaillée par J. Goody. La spatialisation associée à l'écrit et à l'écriture engendre des "possibilités cognitives nouvelles " pour celui qui reçoit mais aussi pour celui qui produit le discours. En se détachant de la linéarisation attachée à l'oral, le scripteur peut revenir sur ce qui est écrit, « procéder à des examens rétrospectifs » (Goody, 1979, p. 221), prendre appui sur ce qu'il a déjà écrit et faire fond sur ce déjà-là du discours pour avancer dans la construction de son texte.

2 Ces appréhensions dichotomiques de l'écriture et de la parole sont potentiellement perturbées par les technologies de l'information et de la communication. La mise au point de logiciels de reconnaissance vocale performants nous amène à reconsidérer la frontière entre l'écriture et la parole. D. Sperber (2002) voit dans ces logiciels l'avènement d'une division du travail scriptural, entre humains et machines, qui devrait conduire à la 
"généralisation de la production orale de textes écrits ». Si par le geste scriptural, on est passé «de la voix à la main» (Goody, 1979, p. 97), quel changement dans la raison graphique sera déterminé lorsqu'on se met à écrire en parlant? L'écriture perd-elle alors une partie de ses vertus ou au contraire gagne-t-elle certaines de la parole ? Qu'en est-il de l'« exercice de rumination constructive » (ibid.) que permet l'écriture?

Nous proposons d'interroger ces questions à travers un dispositif didactique qui, à la faveur de l'utilisation d'un logiciel de reconnaissance vocale (Dragon Naturally Speaking 12.0), prescrit une composition orale comme mise au jour d'un « déjà-là » du texte visé.

Ce dispositif a été construit et mis en œuvre par nos soins au cours de l'année 2014-2015, au sein d'un parcours de master en rédaction professionnelle de l'université d'AixMarseille. Il se caractérise par :

- un contexte d'enseignement-apprentissage d'un savoir-faire en écriture de haut-niveau ;

- une tâche d'écriture très spécifique, celle du résumé ;

- des étudiants déjà aguerris à l'utilisation du traitement de texte, et plus largement à un environnement numérique d'écriture ;

- l'intégration d'un logiciel de reconnaissance vocale à la fois comme outil susceptible de faciliter certaines opérations dans la pratique professionnelle et comme outil d'apprentissage.

5 La composition orale à laquelle nous contraignons les rédacteurs en formation repose sur l'idée d'un gain possible en efficacité dans ce type particulier de tâche d'écriture. Elle présente aussi un intérêt didactique au sens où elle permet de travailler la représentation que les étudiants se font de la tâche à réaliser. Nous faisons l'hypothèse que le fait de passer par une composition orale favorise une concentration de la mobilisation des matériaux linguistiques, et permet de passer, de manière plus rapide, d'un "déjà-là " encore en friche à un « déjà-là » envisagé comme un possible acceptable du texte visé.

6 Le souci de la preuve est délicat à satisfaire en matière didactique dès que les tâches visées sont complexes, et les variables nombreuses et difficiles à neutraliser : il ne s'agit pas ici de proposer un dispositif expérimental qui permettrait de mesurer un gain effectif en rapidité ou en qualité des textes produits, mais d'exposer le dispositif didactique mis en œuvre et de l'étayer en mettant au jour les présupposés et les enjeux sur lesquels il est construit.

\section{Le contexte d'un parcours professionnalisant en écriture de haut niveau}

7 Avatar moderne du scribe ou du rhéteur, le rédacteur professionnel (RP) exerce son activité en indépendant ou en tant que salarié dans des agences de communication, des entreprises, des organismes culturels, des collectivités, où il répond aux missions les plus diverses dans le domaine de l'écrit (papier et numérique) et occupe des postes aux appellations variées (rédacteur-concepteur, rédacteur-réviseur, rédacteur des débats, rédacteur web ou encore chargé de communication écrite). Son savoir-faire est marqué par la complexité (Beaudet, 1999 ; Beaudet \& Clerc, 2008), ce qui amène à parler d'une écriture « experte » ou "de haut niveau » (Beaudet \& Rey, 2015, p. 8). De fait, le RP doit être capable de s'adapter à différents genres, configurations textuelles, visées et supports. Il se trouve en outre dans une situation d'énonciation particulière, une double énonciation imbriquée : il écrit à la place de son client, le mandant, dont il est le truchement, et il doit 
à la fois répondre aux attentes de ce premier lecteur, et à celles du lecteur final, auquel le mandant destine le texte. Cela suppose tout autant une bonne connaissance du destinataire final que du mandant afin d'espérer répondre à l'injonction faite au rédacteur professionnel d'«écrire pour son lecteur» (Labasse, 2009), injonction reformulée de manière plus globale en un «écrire pour être lu » qui permet de ne pas s'arrêter à une vision réductrice du lecteur et de ses compétences (Labasse, 2015, p. 50).

Pour former à une telle complexité, le curriculum dans lequel s'insère notre dispositif mêle apport de connaissances pluridisciplinaires sur l'écrit et l'écriture, innutrition et entrainements nombreux (cf. Pereira, Romain \& Rey, 2015, pour une explicitation des principes de construction du parcours).

Le caractère professionnalisant du cursus et le fait que l'expertise du RP repose sur l'adaptation au lecteur, amènent, encore plus que de coutume en matière d'enseignement-apprentissage de l'écriture, à relativiser la question du bien écrire, et suppose que l'équipe enseignante accepte de se laisser déposséder un tant soit peu de son autorité lorsqu'il s'agit d'évaluer les textes produits par les étudiants. Si un écrit réussi est un écrit adapté au lecteur visé, ce dernier ne peut nullement dans le cadre d'une formation professionnelle se réduire à l'enseignant. Au sein des deux années de master, les occasions d'écriture pour des mandants extérieurs sont ainsi multipliées, et, à défaut, les étudiants sont amenés à être lecteurs des textes de leurs condisciples mais aussi à analyser leurs propres écrits : cette capacité à verbaliser ses choix et à évaluer son propre savoir-faire constitue un élément nodal dans la définition de l'expertise (Beaudet, 1999 ; Schriver, 2012).

Pour s'adapter à leur futur environnement professionnel, les étudiants utilisent de manière courante le traitement de texte : tous doivent avoir un ordinateur portable en cours et les travaux demandés sont rendus sous forme numérique ; c'est aussi le cas d'une grande partie des retours effectués par les enseignants, via les fonctions de révision et de commentaire offertes par le logiciel.

11 Terminons de brosser le contexte général du dispositif en soulignant que les étudiants concernés peuvent être, dès le début des deux années de master, considérés comme ayant des compétences avancées en écriture : ils détiennent à minima une licence en sciences humaines (souvent déjà un master voire une thèse), mais surtout ils doivent manifester, lors de leur candidature d'admission au parcours, un goût pour l'écriture et des aptitudes en la matière. Si cela ne préjuge ni de leur pratique, ni de leurs compétences effectives, ils se perçoivent - sauf candidature fantaisiste ! - comme ayant déjà des capacités d'écriture avérées.

\section{Une tâche d'écriture particulière : le résumé}

\section{L'activité résumante envisagée à l'opposé d'un travail de marquèterie}

Parmi les différentes tâches d'écriture auxquelles les étudiants sont formés, nous nous focalisons ici sur le résumé de documents. Sous des appellations diverses, «l'activité résumante » (Schnedecker, 1991b, p. 65) fait partie de missions potentiellement confiées au rédacteur professionnel. Il peut s'agir de rendre compte de documents isolés en les réduisant ou de proposer des synthèses de différents textes portant sur des thématiques 
communes. Cette tâche n'est souvent qu'un préalable à la rédaction de documents plus complexes : ces résumés peuvent nourrir d'autres documents et fonctionner comme des avant-textes, en s'insérant par exemple dans des rapports ou des livres blancs. Il importe alors que la pratique de la "paraphrase réductrice» (Vigner, 1991, p. 35) devienne une routine pour les rédacteurs en formation.

13 Le résumé a été l'objet d'un enseignement dans le secondaire entre 1969 et 2001, période au cours de laquelle il faisait partie d'un sujet possible à l'EAF, à côté du commentaire et de la dissertation. La présence d'une telle épreuve au baccalauréat répondait au souci d'adapter l'enseignement des lettres à la société de l'information: il s'agissait alors de " former les jeunes générations à maîtriser la quantité sans cesse croissante de données auxquelles elles seraient fatalement confrontées dans leur vie professionnelle et dans leur vie de citoyen » (Charolles, 1991, p. 7). Cette préoccupation rejoint la pratique du résumé dans les missions confiées au rédacteur professionnel, mais l'institution scolaire a transformé cette activité "en un exercice "étriqué" et "calibré" " (Schnedecker, 1991a, p. 5) bien éloigné des usages sociaux variés auxquels le résumé peut être associé.

14 Le résumé scolaire enjoignait de reproduire en réduisant. Les élèves devaient mimer le registre énonciatif du texte d'origine, comme s'ils étaient eux-mêmes auteur de ce texte sans avoir cependant le droit d'utiliser les mêmes mots, si ce n'est pour ceux considérés comme non «traduisibles». À cette injonction contradictoire entre «les exigences de fidélité au contenu et de condensation-reformulation» (Charolles, 1991, p. 10), s'ajoutait une contrainte de longueur qui ne prenait nullement en compte les spécificités des textes de base.

15 M. Charolles a pu ainsi dire qu'il s'agissait, dans la forme figée que l'institution scolaire lui avait conférée, d'une « activité typiquement écrite pratiquement inconcevable à effectuer à l'oral ( (ibid., p. 13). Les processus rédactionnels mis en œuvre lors du résumé étaient, de fait, appréhendés en référence à la «raison graphique » et s'inscrivaient dans les potentialités cognitives ouvertes par l'écriture qui « permet de procéder à des examens rétrospectifs » (Goody, 1979, p. 221). En outre, l'évaluation était effectuée par un enseignant bien plus correcteur que lecteur, ayant connaissance du texte d'origine et jaugeant la production en référence à ce dernier.

16 Dans une telle conception de l'exercice de résumé, le processus rédactionnel écrit s'apparente à un travail de marquèterie qui prend le risque de perdre de vue le tout discursif, le dessin d'ensemble, au profit d'une attention survalorisée aux détails. C'est à l'opposé de la façon dont nous envisageons l'activité résumante pour les rédacteurs en formation. L'objectif est d'apprendre à résumer pour un destinataire qui n'aura pas lu le texte d'origine et qui tirera du résumé des informations pour à son tour écrire ou prendre des décisions.

17 Aussi les consignes d'écriture données dans le cursus de master diffèrent-elles de celles du résumé « scolaire $»^{1}$ :

- la reformulation n'est pas perçue comme une plus-value et le lexique du texte d'origine peut être repris dans un souci de précision et de fidélité ;

- les voix énonciatives doivent être clairement identifiées, le rédacteur se situant dans une position discursive extérieure ;

- l'échelle de réduction est variable.

18 La fidélité aux idées du texte d'origine n'est certes pas évacuée, mais nous gardons à l'esprit que la réduction suppose une lecture-interprétation du texte, que cette lecture 
n'est pas unique et est orientée selon l'usage à venir du résumé2. Le résumé est considéré comme un texte autonome qui doit servir de source d'informations pour un lecteur qui n'aurait pas accès au texte d'origine. C'est alors à l'aune du concept de clarté que les résumés sont évalués, comme peuvent l'être, au sein du cursus, d'autres productions textuelles à visée informative.

\section{Des textes évalués à l'aune du concept de clarté}

19 Le concept de clarté n'est pas récent et la rhétorique antique en faisait déjà, aux côtés de la pureté, de l'élégance et de la convenance, une des conditions principales de l'éloquence. Dans son De oratore, Cicéron (2010, p. 20), par l'intermédiaire du juriconsulte Crassus, la définit de la manière suivante: "Il est évident qu[e les moyens de nous exprimer avec une parfaire clarté] consistent à parler un latin pur et qui exprime d'une façon précise ce qu'on veut énoncer et mettre en lumière, à ne pas employer de périodes trop longues, à ne pas prolonger les métaphores empruntées à la comparaison d'autres objets, à ne pas violer les rapports de temps, à ne pas confondre les personnes, à ne pas embrouiller le plan." Quelques vingt siècles plus tard, cette qualité que l'Arpinate présente comme un aspect élémentaire de l'art oratoire, est une des pierres angulaires de la recherche en rédactologie (Beaudet, 2001). Le souci de mesurer la clarté des textes afin d'agir en retour sur la production de documents accessibles au plus grand nombre, a donné naissance à de nombreux travaux. B. Labasse (1999a, p. 88) distingue deux voies qui ont été empruntées pour mener à bien cette entreprise : celle qui cherche les variables textuelles susceptibles de renseigner sur la lisibilité, et celle qui, s'appuyant sur diverses disciplines académiques, la psychologie, la neurologie, la linguistique, l'informatique, etc., "participe à la compréhension des processus cognitifs qui interviennent dans la production et la réception du discours, l'intégration des informations et leur mémorisation». Cette dernière voie est ambitieuse par son interdisciplinarité et son pari de proposer des modèles de production automatisables de textes lisibles, mais peine à tenir ses « promesses sociétales » du fait même de la complexité de la tâche. La première voie est, quant à elle, en prise avec l'application, s'est essoufflée sur le plan théorique mais exerce une «influence sociétale considérable ». B. Labasse souligne ailleurs (1999b, p. 277) le caractère " assez sommaire » des formules classiques de lisibilité : basées sur la longueur des mots et la longueur des phrases, elles constituent un "indicateur intéressant, bien qu'approximatif, de la facilité de décodage lexico-syntaxique d'un document, mais non de son intelligibilité : un texte peut être facile à lire et n'avoir aucun sens ». L'assimilation de la clarté à la lisibilité est réductrice et les recherches s'orientent souvent vers une attention portée aux environnements cognitifs globaux des destinataires (Cloutier, 2001).

Les étudiants ont été familiarisés avec la clarté à partir d'un article de recherche qui fait le point sur les notions le plus souvent convoquées pour circonscrire ce concept (Beaudet, 2001). Trois composantes sont ici prises en compte pour définir la clarté : la lisibilité, la cohésion et la cohérence ; trois composantes qui font système et se trouvent en tension, une trop grande lisibilité pouvant aller à l'encontre de la cohésion et de la cohérence. Dans un souci d'opérationnaliser le concept pour les besoins de la formation, et sans occulter que la clarté est forcément relative au lecteur, chacune de ces notions a été associée à des marques textuelles ou discursives ${ }^{3}$ pour permettre une mesure certes 
partielle, mais déjà instructive de la clarté d'un texte. Enseignants et étudiants seront amenés à utiliser cet outil pour évaluer les résumés produits au cours de l'année.

\section{Un logiciel de reconnaissance vocale intégré à un dispositif didactique}

$21 \mathrm{Au}$ cours de l'année universitaire 2014-2015, nous avons intégré un logiciel de reconnaissance vocale au dispositif didactique mis en œuvre pour former les étudiants ${ }^{4}$ de première année du master à l'activité de résumé. Il s'agissait à la fois d'initier les étudiants à un outil qu'ils pourraient être amenés à utiliser dans leur vie professionnelle, et d'intégrer ce logiciel comme un médium d'apprentissage, susceptible de les mener vers une plus grande maîtrise dans l'activité de résumé.

Durant le premier semestre, cette activité est travaillée spécifiquement: les étudiants sont confrontés à des tâches de résumé de documents de longueur diverse, notamment des articles scientifiques. Le travail de lecture et d'écriture demandé est étayé par les apports disciplinaires d'autres cours suivis dans le semestre (linguistique textuelle, aspects rhétorique et poétique de la question des genres et registres, premières notions de pragmatique). Les retours et évaluations de l'enseignante chargée du cours sur le résumé n'occultent pas la question de la fidélité au texte d'origine mais privilégient une lecture des résumés comme textes autonomes, à la lumière du concept de clarté, grâce à la grille mise au point avec les étudiants.

Parallèlement, un travail sur la composition orale est effectué via le logiciel d'enregistrement et de traitement audio Audacity. Les étudiants sont amenés à résumer à l'oral des articles scientifiques, à partir de notes écrites succinctes. L'accent est mis sur la projection et le rythme de la voix pour aboutir à un discours intelligible pour l'auditeur. Les étudiants s'entrainent ainsi à la production d'un oral qui emprunte à l'écrit ses normes de construction syntaxique, un oral finalement très " écrit ". Les productions orales font l'objet d'une évaluation par l'enseignante, toujours à l'aune du concept de clarté, mais en y intégrant les éléments propres à la réception d'un discours oral.

$\mathrm{Au}$ second semestre, les étudiants sont initiés à l'utilisation de Dragon: une première demi-journée leur permet se familiariser avec le logiciel et de le paramétrer à leur voix ; lors d'une deuxième demi-journée, il leur est demandé de produire avec ce logiciel le résumé de deux documents. Aucune contrainte de longueur n'est précisée.

Les deux documents à résumer sont extraits d'une enquête publiée par la Société Québécoise de Rédaction Professionnelle (Dumas, 2013) (SQRP). Un des membres de cette association, J. Dumas, a interrogé des enseignants en rédaction professionnelle sur "l'évolution prévisible du métier de rédacteur professionnel au cours des prochaines années ». Chacun des deux textes correspond au compte-rendu de l'entretien entre J. Dumas et deux enseignants autour d'une question: «La rédaction de vulgarisation scientifique exigera-t-elle qu'on soit savant?» pour le premier texte, «La rédaction publicitaire reprendra-t-elle l'initiative?» pour le second. Le choix de ces documents correspond au souci de proposer aux étudiants des textes relativement faciles à résumer pour leur première expérience avec Dragon : la thématique leur est familière, ce qui est censé aider à la compréhension ${ }^{5}$; les textes répondent à une question explicite, leur visée est ainsi manifeste ; l'énonciation est certes polyphonique mais les voix se succèdent sans se chevaucher, J. Dumas commençant par une présentation du contexte et des enjeux de 
la question posée, avant de rapporter successivement les propos des deux enseignants interrogés ${ }^{6}$.

Les documents à résumer sont distribués à la fois sous forme numérique et sous forme papier; les étudiants sont autorisés à souligner ou à surligner les documents papier et même à y prendre quelques notes, mais ils ne sont pas autorisés à produire sous forme manuscrite le résumé qu'ils se contenteraient ensuite de dicter à Dragon; ils doivent dicter au logiciel le résumé tout en le produisant, ils sont donc contraints à une composition orale prenant simplement appui sur le texte d'origine qu'ils conservent devant les yeux, et sur les quelques annotations qu'ils ont souhaité y porter. Pour les besoins de l'exercice, ils ne sont pas autorisés à profiter de toutes les fonctionnalités de Dragon: les révisions par le biais du clavier sont proscrites; seules les commandes orales peuvent être utilisées ; ces révisions ne doivent en outre concerner que des éléments de correction syntaxique ou orthographique (notamment lorsque le logiciel a mal " compris » ce qui a été dicté). Les étudiants sont enjoints d'envisager leur production textuelle orale comme une mouture susceptible d'être évaluée au même titre qu'une production achevée.

Deux semaines après, les résumés transcrits par Dragon font l'objet d'une double évaluation à l'aune de la clarté : en se basant sur la grille organisée autour de la lisibilité, de la cohésion et de la cohérence, chaque étudiant doit tout d'abord évaluer les textes d'un autre étudiant, puis les deux textes qu'il a lui-même produits.

Avant d'observer les évaluations des étudiants, mettons au jour les enjeux de ce dispositif didactique.

\section{Les enjeux du recours à un logiciel de reconnaissance vocale : accepter un premier jet comme un « déjà-là » acceptable du texte visé}

\section{"Écrire n'est pas la seule façon de produire des écrits » (Sperber, 2002)}

Le guide de l'utilisateur de Dragon présente le logiciel comme une aide à la rédaction sur traitement de texte: "Avec Dragon, vous pouvez créer facilement et rapidement vos documents en utilisant des commandes vocales, vous libérant ainsi de la contrainte du clavier7. » Le médium d'écriture est modifié, le geste de taper au clavier est remplacé par la dictée et on peut imaginer toutes sortes d'usage de ce logiciel et différentes façons d'envisager la dictée.

Laissons hors de notre champ de réflexion la dictée à Dragon d'un texte déjà écrit de manière manuscrite, ce qui serait uniquement une façon d'éviter la saisie au clavier, pour nous focaliser sur la dictée d'un texte que l'on est en train d'écrire, de structurer et de mettre en mots. Nous effectuons alors un "acte scriptural », nous produisons un « objet linguistique », sans effectuer d'« acte graphique » (Plane, 2006, p. 33). Le logiciel prend en charge une part de l'activité d'écriture: il gère en l'occurrence les contraintes graphomotrices, « l'acte graphique », mais aussi les contraintes orthographiques, qui font traditionnellement partie de «l'acte scriptural ». 
31 Les potentialités pédagogiques d'un tel outil ont déjà été soulignées pour des scripteurs novices ou présentant des troubles dans l'apprentissage de l'écriture : c'est d'ailleurs à notre connaissance sur ces seuls publics que se focalisent pour l'instant les études évoquant les logiciels de reconnaissance vocale. Ces outils sont utilisés pour alléger le coût cognitif de la tâche d'écriture en libérant le scripteur des opérations de bas niveau, ce qui peut lui permettre de développer ses compétences dans d'autres aspects de la tâche (cf. par exemple Benoit \& Sagot, 2008, ou Despres, 2010).

Ce type d'utilisation n'est pas à exclure pour des scripteurs experts, c'est d'ailleurs sans doute ce que visent les concepteurs du logiciel: en automatisant ainsi certaines opérations de bas niveau, on peut attendre un gain en vitesse et, dans une utilisation non contrainte du logiciel, la dictée peut n'être qu'une façon d'écrire un premier jet soumis ensuite à des révisions plus ou moins importantes, effectuées soit au clavier, soit à l'aide des commandes vocales ${ }^{8}$. Ce peut aussi être un moyen de transformer en écrit lisible et aisément réutilisable, des notes, des impressions, des réflexions que l'on aurait auparavant simplement confiées à un carnet ou à un dictaphone. Cela ne manque pas de renvoyer à des pratiques d'écrivains dictant leurs œuvres à des secrétaires ou à des proches, soit du fait de l'impossibilité d'accomplir eux-mêmes le geste graphique (on pense à J. Milton dictant à ses filles et à J. L. Borges dictant à son assistante), soit par choix révélateur sans doute de leur rapport à l'écriture (A. Cohen dictant ses romans aux femmes de sa vie ${ }^{9}$, Solal à Yvonne Imer, Belle du Seigneur à Bella ; les notes étaient ensuite dactylographiées, puis largement reprises, éventuellement lors d'une nouvelle dictée). Le fait de composer ainsi oralement peut tout autant se trouver associé à la volonté de conserver la trace des hésitations de l'oral, ou de pallier sa lenteur d'écriture et de bénéficier de la rapidité d'un secrétaire transcrivant en sténographie, et permettant ainsi d'écrire à la vitesse de la pensée, ou du moins de la parole.

Le logiciel de reconnaissance vocale met ainsi à la portée de tous un secrétaire, l'aspect affectif de la relation en moins (ce qui peut, selon le scripteur ou les moments, être perçu comme un manque ou un bienfait). Pariant sur l'amélioration des logiciels de reconnaissance et de lecture vocales, et dissociant l'écrit de l'écriture, D. Sperber annonçait en 2002 la disparition de l'écriture tout en promettant encore de beaux jours à la lecture: "L'écriture est pour l'essentiel un coût payé pour pouvoir profiter de la lecture. C'était et c'est encore un coût presque inévitable, mais plus pour très longtemps. Dès que la technologie permettra de voir sa parole correctement transcrite au fur et à mesure de son déroulement et de modifier le texte transcrit par des instructions orales (et sans doute aussi par des gestes de pointage et de sélection de texte), l'écriture ne présentera plus aucun avantage qui en justifie le coût. En revanche, se faire lire un texte à haute voix par une machine est rarement préférable à la lecture personnelle. »

Notre propos n'est cependant pas de mettre les étudiants dans une situation où ils pourraient se passer de l'écriture: il s'agit pour ces scripteurs aguerris, qui ont déjà automatisé une large partie des procédures de bas niveau, d'user du logiciel de reconnaissance vocale comme d'un moyen de travailler la maitrise de l'écrit pour certains mandats d'écriture. 


\section{Composer oralement : tension entre linéarisation de l'oral et spatialisation de l'écrit}

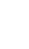
textuelle qui s'inscrive pleinement dans la linéarité de l'oral et qui limite le recours à la spatialisation de l'écrit. L'oralisation, par le caractère irrémédiable de sa réception, peut à priori apparaître comme un obstacle à la richesse du processus de rédaction, la spatialisation de l'écrit et la dissociation du temps de la production et de celui de la réception autorisant allers-retours, reprises, reformulations, transformations, ajouts ou suppressions. La tension entre linéarité de l'oral et spatialisation de l'écrit est ici à envisager selon un gradient mesurant le caractère «définitif » que l'on attribue à la production discursive. Le médium d'écriture, mais surtout la façon dont on en fait usage, déterminent ce caractère, et le mouvement irréversible de l'oral contamine alors l'écrit selon des degrés divers. En contraignant les étudiants à composer oralement sans revenir sur leur production textuelle, nous souhaitons qu'ils s'éloignent de l'impunité du geste scriptural qu'ils vivent à la faveur du traitement de texte, et que leur production textuelle soit «contaminée » par le caractère irrémédiable de l'oral. Nous ne suggérons évidemment pas que le processus d'écriture ne serait alors qu'un flux unidirectionnel et nous souscrivons à la représentation d'une production du texte où les phénomènes en jeu correspondent à « un ensemble de plaques [...] glissant l'une sur l'autre à des vitesses différentes » (Plane, Alamargot \& Lebrave, 2010, p. 17). Nous n'ouvrons pas la boite noire de la production textuelle, nous contraignons à une composition orale qui ne prétend nullement n'être qu'un aspect de la production textuelle; ainsi le processus de planification et celui de textualisation sont-ils déjà à l'œuvre dans les quelques notes que les étudiants peuvent prendre sur les documents papier; et ils le sont encore lors de la phase de dictée, tout comme lors de l'écriture d'un texte.

38 Dans l'activité résumante, nous avons vu que le processus rédactionnel écrit peut s'apparenter à un travail de marquèterie qui prend le risque de perdre de vue le tout 
discursif, le dessin d'ensemble, au profit d'une attention survalorisée aux détails. Par analogie avec l'entrée dans le langage écrit qui nécessite que le langage oral soit suffisamment placé (Morais \& Robillart, 1998), nous faisons l'hypothèse que l'apprentissage d'une oralité de haut niveau peut être mis au service de la production rédactionnelle de haut niveau. Passer par une composition orale exige de mettre au jour une représentation globale du texte à produire, portée par une attention privilégiée à la visée du discours à résumer. La composition orale est exigeante parce qu'elle peut s'apparenter à un saut dans le vide, mais elle est facilitante parce qu'elle préserve de l'émiettement et de la dispersion. Le fait d'entrer dans le discours oral favorise une concentration de la mobilisation des matériaux linguistiques, et le recours au logiciel de reconnaissance vocale permet de passer, de manière plus rapide, d'un « déjà-là » encore en friche à un « déjà-là » envisagé comme un possible acceptable du texte visé.

Le fait que l'exercice concerne une activité de résumé n'est pas anodin : le « déjà-là » de la composition orale s'appuie sur un « déjà-là » que le scripteur va s'approprier, le textesource. L'existence même de cet avant-texte, dont la disponibilité est grande, doit conduire les apprentis rédacteurs à considérer la tâche de résumé comme plus simple que les autres travaux d'écriture et non comme la réalisation d'une œuvre qu'ils pourraient améliorer à l'infini. Loin d'être un renoncement, accepter le premier jet comme un texte lisible et communicable, nous permet de travailler sur l'idée que la pratique d'écriture n'a pas à être homogène, et qu'en fonction des tâches et des visées du document à produire, l'engagement du scripteur et son exigence vis-à-vis de sa production doivent être modulés.

En l'occurrence, en dépit de la complexité de la tâche pour un grand nombre de scripteurs, produire un résumé qui informe sur le contenu d'un autre texte ne doit pas requérir autant de brio qu'une autre tâche. Les étudiants expérimentent l'idée qu'« un texte définitif est bien souvent une ébauche prise sur le fait» (Senges, 2015, p. 127) : nous plions certes ici la pensée de ce romancier contemporain à notre objet ; il évoque alors la création littéraire et déclare profiter de "l'impunité du brouillon » pour produire ce qui sera, sans qu'il ne le sache au départ, le texte définitif. Cela rejoint cependant notre souhait d'amener les étudiants à expérimenter l'absence de différence de nature entre ce qu'ils pourraient percevoir comme un brouillon et ce qu'ils nommeront texte définitif.

L'extrême attention à laquelle la composition orale les conduit peut s'imaginer sans le recours à l'oral, et nous pourrions leur demander de produire le résumé sans revenir sur ce qu'ils écrivent, en saisissant au traitement de texte ou en écrivant de manière manuscrite ${ }^{10}$. Mais le recours à Dragon et la linéarité associée à l'oral facilitent ce saut dans le vide.

Le dispositif didactique contraint à une utilisation extrême du logiciel, une utilisation qui ne sera vraisemblablement pas celle que les étudiants auront dans leur vie professionnelle. Il s'agit ici non pas simplement de former les étudiants au logiciel, mais de l'utiliser comme un outil dans l'apprentissage. En allant ainsi jusqu'au bout des potentialités de Dragon, nous visons à ouvrir des potentialités dans les pratiques d'écriture de ces futurs professionnels. Tout en gardant à l'esprit le caractère idiosyncrasique de l'écriture, nous espérons que les étudiants s'essaient ici à des pratiques scripturales extrêmes, pour élargir la palette de leurs compétences ${ }^{11}$. 


\section{Observation de l'évaluation réalisée par les étudiants}

43 Dans le cadre du présent article, nous ne procèderons pas à une analyse approfondie des résumés produits, même s'il nous paraîtrait intéressant d'observer notamment les procédés de textualisation à l'œuvre dans cette composition orale. Nous nous contenterons ici de sélectionner quelques éléments en lien avec l'évaluation produite par les étudiants.

Cette évaluation constitue une étape dans le dispositif didactique et non une mesure de sa pertinence en termes de gain immédiat sur les productions textuelles. Le dispositif exposé ne constitue pas un dispositif expérimental et il ne s'agissait pas d'espérer créer des conditions pour mesurer un progrès en termes de rapidité ou une amélioration qualitative des textes produits avec Dragon ${ }^{12}$. Si le souci de la preuve taraude l'enseignantchercheur expérimentant un dispositif, dès lors que la tâche est complexe, monter un protocole qui permette de neutraliser les variables de manière convaincante apparait souvent comme particulièrement lourd, voire comme une gageure. L'évaluation des résumés par les étudiants nous renseigne cependant sur la pertinence du dispositif en termes de représentation de la tâche d'écriture à accomplir. Le dispositif didactique leur donne à lire le texte produit par la composition orale : les apprentis scripteurs observent ce «déjà-là » de la production textuelle, "déjà-là » qui constitue un stade de la représentation mentale du texte en train de se construire dans l'activité résumante.

Rappelons qu'il est demandé à chaque étudiant, deux semaines après la réalisation de la "composition orale», d'évaluer tout d'abord deux résumés produits par un autre étudiant, puis les deux résumés qu'il a lui-même produits. L'évaluation se fait à l'aide de l'outil construit à partir de l'article de C. Beaudet (2001) faisant le point sur le concept de clarté : une grille qu'ils ont l'habitude de pratiquer, et qui organise les caractéristiques discursives selon trois dimensions, la lisibilité, la cohésion, et la cohérence (cf. note 3 du présent article). Les étudiants sont rompus à l'idée que la clarté du texte est le résultat de caractéristiques hétérogènes et multiples; ils ont été sensibilisés au fait qu'une trop grande lisibilité, si on ne la mesure qu'en termes d'éléments linguistiques, entraîne des difficultés de cohésion et de cohérence. Ils doivent à la fois commenter les résumés et procéder à une évaluation chiffrée (une note sur 100, résultat de notes attribuées à chacune des caractéristiques discursives identifiées).

Volontairement, les critères d'évaluation ne font pas mention de la conformité au textesource : on fait le pari que le résumé doit être évalué comme un texte qui n'est pas lu après le document d'origine. Dans les commentaires des étudiants, nous verrons que ce point de référence est tout de même présent.

D'un point de vue général, les notes attribuées par les étudiants sont bonnes, voire très bonnes (tableau 1).

Tableau 1. Évaluation chiffrée globale des résumés par les étudiants

\begin{tabular}{|l|l|l|l|l|l|l|}
\hline & $\begin{array}{l}\text { Résumés de } \\
\text { l'étudiant A }\end{array}$ & $\begin{array}{l}\text { Résumés de } \\
\text { l'étudiant B }\end{array}$ & $\begin{array}{l}\text { Résumés de } \\
\text { l'étudiant C }\end{array}$ & $\begin{array}{l}\text { Résumés de } \\
\text { l'étudiant D }\end{array}$ & $\begin{array}{l}\text { Résumés de } \\
\text { l'étudiant } \mathrm{E}\end{array}$ & $\begin{array}{l}\text { Résumés de } \\
\text { l'étudiant } \mathrm{F}\end{array}$ \\
\hline
\end{tabular}




\begin{tabular}{|l|l|l|l|l|l|l|}
\hline $\begin{array}{l}\text { Note attribuée } \\
\text { par l'étudiant } \\
\text { ayant produit } \\
\text { les résumés }\end{array}$ & 77 & 69 & 80 & 60 & 78.5 & 68.5 \\
\hline $\begin{array}{l}\text { Note attribuée } \\
\text { par un autre } \\
\text { étudiant }\end{array}$ & 76 & 85 & 88 & 75 & 81 & 84.5 \\
\hline
\end{tabular}

\section{Tableau 2. Détail des l'évaluation chiffrée des résumés par les étudiants}

\begin{tabular}{|l|l|l|l|l|l|l|l|l|}
\hline & & A & B & C & D & E & F & Moyenne \\
\hline \multirow{4}{*}{$\begin{array}{l}\text { Note attribuée par l'étudiant ayant } \\
\text { produit les résumés }\end{array}$} & Lisibilité / 32 & 24 & 24 & 20 & 13 & 22,5 & 19 & $20,41 / 32$ \\
\cline { 2 - 11 } & Cohésion / 34 & 27 & 28 & 27 & 24 & 29 & 28,5 & $27,25 / 34$ \\
\cline { 2 - 11 } & Cohérence / & 26 & 17 & 33 & 23 & 27 & 21 & $24,5 / 34$ \\
\hline \multirow{3}{*}{ Note attribuée par un autre étudiant } & Lisibilité / 32 & 30 & 26 & 24 & 22,5 & 25 & 23 & $25,08 / 32$ \\
\cline { 2 - 11 } & Cohésion / 34 & 29 & 30 & 30 & 27 & 30 & 31,5 & $29,58 / 34$ \\
\cline { 2 - 11 } & Cohérence / & 17 & 29 & 34 & 25,5 & 26 & 30 & $26,91 / 34$ \\
\hline
\end{tabular}

Les étudiants auraient à priori aimé retoucher leur production et ne pas s'en tenir à ce « déjà-là », or l'évaluation conjointe les amène à conclure à la clarté de leurs résumés : ces résultats les invitent à percevoir dans la composition orale une pratique qui ne mettrait pas en péril la clarté de la production.

À une exception près (résumés de l'étudiant A), les notes attribuées aux résumés produits par l'autre étudiant sont meilleures que lors de l'autoévaluation. On peut faire l'hypothèse que les étudiants, au sein d'une promotion réduite où les travaux de groupe sont nombreux et contribuent à tisser des liens, font preuve de bienveillance à l'égard de leur camarade. On peut aussi y voir le reflet de la grande exigence qu'ils manifestent à l'égard de leurs propres travaux.

Si l'on observe plus précisément le détail de l'évaluation chiffrée en s'intéressant aux notes attribuées à chacune des trois composantes (lisibilité, cohésion, cohérence), on relève que c'est la lisibilité qui pèche (tableau 2).

Les commentaires précisent notamment que la terminologie de spécialité contribue à diminuer l'indice de lisibilité (ce qui est dû à la thématique des textes de base et peut difficilement être évité), mais la longueur des phrases joue également. Les étudiants constatent ainsi que la composition orale ne les amène pas forcément à produire des textes aux phrases par trop «simplistes ». En outre la cohésion obtient les meilleurs scores: la composition orale n'a pas empêché la présence des marques de cohésion, réputées « donn[er] du volume à la linéarisation du texte écrit » (Favart, 2005, p. 306). 
Il est intéressant de souligner la présence de commentaires négatifs portant sur la cohérence et expliquant ce qui est perçu comme un défaut, par l'organisation du textesource: "organisation des idées assez anarchique (calquée sur celle des documents d'origine) » ou encore « enchaînement des idées qui suit celui des articles d'origine (et qui est donc incohérent)» (sic). Cela porte à croire que si les étudiants étaient passés par un mode de composition "classique", "écrit ", ils auraient eu à cœur d'améliorer l'organisation des idées des textes d'origine, s'appliquant ainsi au travail de marquèterie dont nous parlions plus haut, et proposant finalement bien plus qu'un résumé. La composition orale imposée les oblige à laisser affleurer davantage l'avant-texte et à considérer que le premier jet du résumé, qui n'a pas à se préoccuper d'améliorer l'organisation des idées du texte d'origine, est déjà un texte acceptable.

Penchons-nous, à titre d'illustration du travail langagier effectué, sur les résumés du premier texte produits par deux étudiants (étudiants A et E).

La visée et l'organisation du texte de départ sont argumentatives : J. Dumas et les deux enseignantes interrogées, $\mathrm{N}$. Kinnard et $\mathrm{L}$. de Serres, répondent de manière nuancée et étayée à la question "La rédaction de vulgarisation scientifique exigera-t-elle qu'on soit savant? » Résumer ce texte suppose que les étudiants aient perçu le tout discursif, soient capables de trier dans ce déjà-là les matériaux à leur disposition pour construire un texte autonome, à même d'informer un lecteur qui n'aurait pas connaissance du texte de base, un lecteur dont le « déjà-là » n'englobe pas le texte d'origine. Les choix effectués par les deux étudiants pour y parvenir sont divergents.

\section{Résumé produit par l'étudiant $\mathrm{A}$}

La rédaction de vulgarisation scientifique exigera-t-elle qu'on soit savant?

Les disciplines scientifiques ont chacune leur jargon, leurs outils et leur logique. Ce sont des domaines clos et difficiles d'accès pour des non-initiés. Cependant pour qu'un savoir global progresse, il doit se déployer. Le vulgarisateur scientifique doit faire le lien entre le savoir établi et l'envie de connaître en accordant la priorité au destinataire. Le travail de vulgarisation est une condition essentielle à la propagation et au rayonnement du savoir. Il est donc question de savoir si les rédacteurs professionnels doivent être eux-mêmes spécialisés pour la rédaction de vulgarisation scientifique.

Dans le processus de vulgarisation, il est nécessaire de cibler correctement le public. Il existe une multitude de publics et un seul document ne peut pas tous les informer. Mais quel que soit le public ciblé, le texte doit être rédigé de manière à ce que son lecteur se sente valorisé. Ceci est la condition d'une vulgarisation réussie. Pour Nathalie Kinnard, la vulgarisation scientifique a un avenir certain. En effet, les médias relayent de plus en plus les savoirs scientifiques. Cette visibilité encourage l'intérêt du public. Les chercheurs sont de plus en plus sensibles à l'importance de la vulgarisation et réclament le savoir-faire de rédacteurs spécialisés. En effet, la vulgarisation présente certains enjeux pour les chercheurs. Ceux-ci, s'ils mettent leurs connaissances scientifiques à la portée des non-spécialistes, pourront plus facilement obtenir des subventions et mettre en avant leur travail. Les chercheurs doivent prendre conscience que la vulgarisation scientifique ne constitue pas un rabaissement mais une mise en relation avec un public plus large. Nathalie Kinnard explique qu'un bon vulgarisateur doit être capable de se mettre dans la peau de quelqu'un qui ne connaît pas le sujet. Selon elle, il y a quatre qualités requises pour un vulgarisateur : une bonne maitrise de la langue, une bonne culture générale, une vive curiosité et une facilité à expliquer. Le rédacteur aura réussi son travail de vulgarisation s'il arrive à maintenir l'attention de son lecteur.

Pour Linda de Serres, le vulgarisateur doit pouvoir naviguer facilement entre plusieurs disciplines différentes et sera un esprit savant en 2020. D'après elle, la 
vulgarisation scientifique constitue une spécialisation difficile mais prometteuse pour les rédacteurs professionnels. Seuls les rédacteurs possédant des connaissances interdisciplinaires variées tireront leur épingle du jeu.

\section{Résumé produit par l'étudiant $\mathrm{E}$}

Ce texte est extrait d'un ouvrage de Jean Dumas, membre d'honneur de la SQRP. Intitulé «la rédaction de vulgarisation scientifique exigera-t-elle qu'on soit savant?", il fait intervenir Nathalie Kinnard et Linda de Serres, respectivement rédactrice scientifique et chercheuse.

Dumas commence par déplorer l'isolement des différents domaines de connaissance, prônant la multiplication des passerelles. Cette tâche incombe pour lui au vulgarisateur scientifique, professionnellement entraîné. Ce dernier fait en sorte que le lecteur se sente toujours valorisé, considéré comme un adulte capable de comprendre. Dumas s'interroge cependant sur la nécessité pour ledit vulgarisateur de se spécialiser.

Nathalie Kinnard est très optimiste quant à l'avenir de la vulgarisation, que ce soit dans les médias ou par son recours par les chercheurs via les rédacteurs spécialisés. Un travail "en langage accessible» se propagera plus aisément et aura plus de retombées que s'il est écrit en langage expert. Cette tendance va croissante selon Kinnard, qui considère la vulgarisation comme une mise en relation plutôt qu'un rabaissement. La rédactrice termine son propos en résumant les quatre exigences du métier de vulgarisateur : français de qualité, vaste bagage culturel, vive curiosité et faciliter à expliquer.

Linda de Serres, elle, insiste sur l'éclatement irrémédiable de la culture scientifique; le vulgarisateur se fera alors débroussailleur. La tendance sera alors à la multicompétence plutôt qu'à la surspécialisation. D'où l'importance de pouvoir naviguer à l'aise entre des domaines en apparence non reliés; le reste de son intervention consiste en 20 conseils au futur vulgarisateur scientifique.

Dans sa conclusion Dumas insiste sur le fait que seuls des rédacteurs aptes à faire voir les ponts entre plusieurs disciplines sauront laisser leur marque, forts d'une compétence assurée et d'une culture vaste et rigoureuse. (cf. tableau 2), cette cohésion n'est cependant pas construite grâce aux mêmes outils et ne fait pas appel au même type de structuration. Les deux étudiants choisissent de conserver l'ordre d'apparition des propos du texte d'origine, mais l'étudiant A privilégie une organisation d'ensemble argumentative alors que l'étudiant $\mathrm{E}$ met en avant l'organisation temporelle du texte d'origine.

L'étudiant $\mathrm{E}$ donne de la cohésion à son texte par des marqueurs d'intégration linéaire temporels (« Dumas commence par », « la rédactrice termine son propos », « « le reste de son intervention consiste en », «Dans sa conclusion Dumas insiste sur ») : il rend ainsi compte de l'organisation des voix énonciatives qui se succèdent dans le texte de base ${ }^{13}$. Domine pour cet étudiant le souci de décrire le document d'origine plus que de proposer une lecture interprétative du discours argumentatif qui y est construit. Dans l'évaluation de son texte, l'étudiant souligne que les connecteurs sont "insuffisants" et que "les idées » du texte de base ont été repérées « sans forcément s'en emparer »; il conclut que l'« intention informative du texte » est « ratée », non rendue par le résumé.

L'étudiant A n'utilise quant à lui pas de marqueurs d'intégration linéaire temporels, ce qui fait d'autant plus ressortir les connecteurs logiques qui organisent le texte. Cette tendance à privilégier le schéma argumentatif se fait en partie ici au détriment de l'identification des voix énonciatives, l'étudiant nommant les deux enseignants interrogés mais ne faisant pas mention de J. Dumas. 
60 Ces choix divergents dans la façon d'assurer la cohésion du résumé peuvent difficilement être évalués de manière absolue: dans une situation professionnelle, l'objectif de l'activité résumante peut naviguer entre le souci de donner à entendre une argumentation et le souci de rendre compte de la répartition des voix énonciatives ${ }^{14}$.

61 Ce qui nous importe ici est que la production du résumé via le logiciel de reconnaissance vocale n'a pas empêché les étudiants d'assurer l'organisation du propos pour le lecteur à venir.

\section{Conclusion}

Dans la tâche de résumé, l'engagement dans l'écriture peut facilement être moindre du fait de la présence d'un avant-texte explicite qui assure la mise à disposition de matériaux linguistiques. Cela a été pour nous l'occasion de faire expérimenter aux étudiants un mode de composition différent : le dispositif didactique a astreint les étudiants à écrire en parlant et ils ont appréhendé un "déjà-là » de l'écrit dans la production orale. Ce qu'ils auraient pu ne considérer que comme une production en friche, s'est avéré être un texte tout-à-fait recevable en termes de clarté.

63 Si l'expertise du rédacteur professionnel passe par sa capacité d'adaptation, au genre, à la visée, à ses lecteurs, il nous semble également important de tirer toutes les conséquences de la relativité de la norme lorsqu'il s'agit de décider de la qualité d'un texte. Une écriture de haut niveau est aussi une écriture qui adapte ses outils, sa jauge et son niveau d'exigence à la tâche d'écriture et à la finalité du texte à produire. Adopter une norme absolue du bien écrire en toutes circonstances risque de conduire certains scripteurs à polir l'objet linguistique que constitue leur texte en ayant comme seul terme le délai de livraison, à l'enseignant pour l'étudiant, au mandant pour le professionnel ; le logiciel de traitement de textes ne fait en outre qu'amplifier cette tendance. Nous n'avons nullement l'ambition ou même le désir de faire adopter par tous les étudiants, pour tout type de tâche d'écriture, la composition orale et le recours à Dragon; il s'agit en revanche d'ouvrir des potentialités nouvelles dans leurs compétences.

\section{BIBLIOGRAPHIE}

ANIS, J. (1995). « Le traitement de texte : écriture ou méta-écriture ? Repères 11, p. 15-27.

BEAUDET, C. (1999). « Les compétences linguistiques et discursives du rédacteur professionnel : un ensemble à circonscrire ». In : Guével, z. \& Clerc, I. (éds), Les professions langagières à l'aube de l'an 2000 : recherches pédagogiques et linguistiques en traduction, rédaction et terminologie. Québec: CIRAL, Faculté des lettres, Université Laval, p. 3-18.

- (2001). «Clarté, lisibilité, intelligibilité des textes : un état de la question et une proposition pédagogique ». Recherches en rédaction professionnelle 1, p. 5-16.

BEAUDET, C., \& CLERC, I. (2008). « Enseigner la rédaction au Québec : Quels fondements disciplinaires? Quelle reconnaissance institutionnelle?». Actes du colloque De la France au 
Québec : l'écriture dans tous ses états. Université de Poitiers, 12-15 novembre 2008. [Actes de colloque électroniques : http://www.ecritfrancequebec2008.org/].

BEAUDET, C. \& REY, V. (éds) (2015). Écritures expertes en questions. Aix-en-Provence : Presses universitaires de Provence.

BENOIT, H. \& SAGOT, J. (2008). « L'apport des aides techniques à la scolarisation des élèves handicapés ». La nouvelle revue de l'adaptation et de la scolarisation 43, p. 19-26.

CHAROLLES, M. (1991). « Le résumé de texte scolaire. Fonctions et principes d'élaboration ». Pratiques 72, p. 7-32.

CICÉRON. (2010) [I ${ }^{\text {er }}$ siècle av. J.-C.]. De l'orateur. Trad. par E. Courbaud et H. Bornecque, Paris : Les Belles Lettres.

CLOUTIER, F. (2001). « La clarté : pour qui ? » Recherches en rédaction professionnelle 1, p. 46-54.

COHEN, A. (1993). Euvres. Paris : Gallimard.

DESPRES, G. (2010). « Les aides techniques au collège auprès d'adolescents avec des troubles des apprentissages ». Développements 6, p. 43-52.

DUCROT, O. \& SCHAEFFER, J.-M. (1995). Nouveau dictionnaire encyclopédique des sciences du langage. Paris : Seuil.

Dumas, J. (2013). La rédaction professionnelle à l'horizon de 2020. En ligne : http:/enquete.sqrp.org.

FAVART, M. (2005). « Les marques de cohésion : leur rôle fonctionnel dans l'acquisition de la production écrite de texte ». Psychologie française 50, p. 305-322.

GooDy, J. (1979) [1977]. La raison graphique. La domestication de la pensée sauvage. Trad. de l'anglais par J. Bazin \& A. Bensa, Paris : Éd. de Minuit.

GRÉGOIRE, P. \& KARSENTI, T. (2013). « Le traitement de texte et la qualité de l'écriture d'élèves québécois du secondaire ». Éducation et Formation e-298-03, p. 9-28.

LABASSE, B. (1999a). « La lisibilité rédactionnelle : fondements et perspectives ». Communication et langages 121, p. 86-103.

- (1999b). « Lisibilité ». In : Combier, M. \& Pesez, Y. (éds), Encyclopédie de la chose imprimée : du papier à l'écran. Paris : Retz, p. 276-277.

- (2009). «L'écrit professionnel : ambiguïtés et identités d'un objet académique ». Pratiques $143 / 144$, p. 233-248.

- (2015). « Les déterminants cognitifs et sociaux de l'adéquation communicationnelle ». In : Beaudet, C. \& Rey, V. (éds), Écritures expertes en questions. Aix-en-Provence : Presses universitaires de Provence, p. 39-71.

MAINGUENEAU, D. (1991). L'analyse des discours. Paris : Hachette.

MORAIS, J. \& ROBILLART, G. (dirs) (1998). Apprendre à lire. Paris : CNDP et O. Jacob.

PEREIRA, M.-E., ROMAIN, C. \& REY, V. (2015). « L'écriture professionnelle : un bel enjeu didactique au croisement de la littérature, de la rhétorique et de la linguistique ». In : Beaudet, C. \& Rey, V.

(éds), Écritures expertes en questions. Aix-en-Provence : Presses universitaires de Provence, p. 253-264.

PLANE, S. (2004). « Quels modèles pour analyser la production d'écrit sur traitement de texte ? Les contraintes comme outil d'analyse et d'intervention ». Linx 51, p. 75-89. 
- (2006). « Médium d'écriture et écriture littéraire ». Le français aujourd'hui 153, p. 33-40.

Plane, S., ALAmargot, D. \& LebraVe, J.-L. (2010). « Temporalité de l'écriture et rôle du texte produit dans l'activité rédactionnelle ». Langages 177(1), p. 7-28.

ROUSSEAU, J.-J. (1990) [1781]. Essai sur l'origine des langues. Paris : Gallimard.

SCHNEDECKER, C. (1991a). « Résumés ». Pratiques 72, p. 3-6.

- (1991b). «Résumés : gammes d'activités ». Pratiques 72, p. 55-90.

SCHRIVER, K. (2012). « What we know about Expertise in Professional Communication ». In :

Berninger, V. W. (éd.), Past, Present, and Future Contributions of Cognitive Writing. New York : New

York Psychology Press, p. 275-312.

SENGES, P. (2015). « Des ébauches prises sur le fait ». Littérature 178, p. 127-142.

SPERBER, D. (2002). «L'avenir de la lecture et de l'écriture ». Texte écrit pour le colloque virtuel text-e, 15octobre 2001-fin mars 2002. En ligne : http://www.dan.sperber.fr/.

VIGNER, G. (1991). «Réduction de l'information et généralisation : aspects cognitifs et

linguistiques de l'activité de résumé ». Pratiques 72, p. 33-54.

\section{NOTES}

1. Mais un des éléments de la formation consiste à souligner que les pratiques sociales sont fluctuantes et qu'en fonction des entreprises, les demandes seront variées, les appellations aussi. Telle entreprise nommera résumé ce qu'une autre appellera synthèse... d'où l'importance de l'adaptation et de la capacité à analyser la demande. Pour les épreuves de concours ou d'examen proposant une activité résumante, la répartition terminologique entre résumé et synthèse se base souvent sur le type de supports : le résumé est produit à partir d'un texte unique et la synthèse à partir de documents multiples. C'est par exemple le cas pour le concours de recrutement de professeur documentaliste, où une "note de synthèse " est demandée à partir d'un dossier de plusieurs documents. Or, dans le cadre du parcours de master qui nous occupe, il ne s'agit pas de former les étudiants à une épreuve de concours définie par un texte officiel, il s'agit de les former à s'adapter, dans le monde professionnel, à des demandes formulées diversement en fonction des locuteurs, sans qu'aucune autorité terminologique ne puisse être invoquée.

2. Au fil des deux années de formation, l'activité de résumé, à côté des travaux spécifiquement orientés vers son apprentissage, prend place au sein de genres variés produits par les étudiants, par exemple lors de l'écriture de critiques d'œuvres, où le résumé - partiel - de l'intrigue doit être intégré à un texte à visée argumentative, la critique devant inciter à lire ou à ne pas lire l'œuvre.

3. Pour la lisibilité : variables lexicales et morphosyntaxiques, «la longueur des mots, la familiarité du vocabulaire, la répartition entre mots concrets et abstraits, la complexité et la forme des phrases » (Beaudet, 2001, p. 7). Pour la cohésion : « l'ordre normatif des mots dans la phrase et le respect des règles d'accord, la distribution (raisonnée) des temps verbaux, les connecteurs, les phénomènes d'anaphorisation, de renominalisation, les marqueurs d'intégration linéaire (temps, espace, progression; Maingueneau, 1991, p. 217), les marqueurs configurationnels (paragraphes, organisateurs métadiscursifs)» (ibid., p. 9). Pour la cohérence : « l'intention [du texte], son topic ou sujet (thème + prédicat), l'interrelation des parties du texte en regard du développement du sujet ou du topic (principe de continuité référentielle), l'identification, la caractérisation et la stabilisation des voix de l'énonciation (énonciateur et énonciataire) » (ibid.) 
4. La promotion en présentiel est composée de six étudiants. Six autres étudiants suivent la formation en ligne mais nous n'avons pas intégré pour l'instant l'utilisation de Dragon au parcours à distance.

5. Le fait de travailler ainsi, de manière incidente, sur la question de l'écriture professionnelle n'est pas isolé dans le parcours : cela répond au souci de permettre un recul réflexif sur la pratique d'écriture et de construire une identité professionnelle en prenant connaissance de la façon dont le métier est perçu, en l'occurrence par une organisation professionnelle associée à des universitaires.

6. N. Kinnard et L. De Serres pour le premier document, J. Desautels et D. Baillargeon pour le second.

7. Dragon, Guide de l'utilisateur, 2012, Nuance Communications, Inc., p. 46.

8. L'utilisation de ce type de logiciel est déjà répandue dans certaines professions, chez les médecins par exemple.

9. "Ces dictées de chaque soir, c'était aussi, peut-être, une manière de ne jamais nous ennuyer ensemble. [...] Lorsqu'on s'est tout dit, dicter un livre est une façon merveilleuse d'avoir encore à se dire, encore à commenter ensemble. Et puis c'est aussi une façon d'offrir. La bien-aimée se réjouissait du don dicté de chaque soir et elle m'en chérissait. Aurais-je eu envie d'écrire mes livres sans les merveilleuses de ma vie ? Je ne crois pas. » (Cohen, 1993, p. 1125)

10. Cf. P. Senges (2015, p.130) expliquant ce qu'il s'impose dans son écriture littéraire manuscrite : «je m'interdis toute rature - mes manuscrits sont anti-flaubertiens, dépourvus du moindre pâté, tristement impeccables. Je m'oblige à avoir cette écriture nette et à écrire les phrases les plus longues possibles, non pas par souci de la prouesse, mais pour aller au-delà de mes forces, pour aller au-delà du premier jet et de l'idée première: de fait, la phrase se compliquant, au cours des digressions et des subordonnées, m'oblige à être plus vigilant que nature, elle m'oblige en quelque sorte à m'échapper de moi-même (pas trop tôt) et, surtout, à m'éloigner de mes premières intentions, quitte à les trahir. "

11. Nous pourrions appeler ce genre de dispositif un OURÉPO, un OUvroir de RÉdaction POtentielle.

12. D'autant qu'il est raisonnable de penser que, comme pour le logiciel de traitement de texte (cf. Grégoire \& Karsenti, 2013), l'utilisation de Dragon doit être automatisée pour que le scripteur en tire des bénéfices mesurables.

13. Apparaît ici une difficulté inhérente au document de base, qui est déjà une reformulation résumante d'autres propos : cette strate antérieure, qui n'est accessible qu'à travers le document de base, apparaît pour cet étudiant comme un élément à prendre en compte, et le choix de mettre en évidence l'organisation linéaire du texte est sans doute lié à l'épaisseur temporelle du feuilleté énonciatif.

14. Les quatre autres étudiants ont privilégié l'attention au schéma argumentatif.

\section{RÉSUMÉS}

La mise au point de logiciels de reconnaissance vocale performants permet d'écrire en parlant et amène à reconsidérer les frontières de l'écrit et de l'oral. Cet article questionne l'apport d'un tel outil dans le cadre d'un parcours de master en rédaction professionnelle, pour une tâche d'écriture spécifique, le résumé. Contraints à une composition orale sans repentir, les étudiants 
ont réalisé un acte scriptural inscrit pleinement dans la linéarité de l'oral et limitant le recours à la spatialisation de l'écrit. L'évaluation par les étudiants de leurs propres productions et de celles de leurs pairs, à l'aune du concept de clarté, permet de conclure que, pour l'activité résumante qui s'appuie sur un avant-texte explicite, la composition orale n'a pas mis en péril la clarté du texte produit: le «déjà-là » du texte dans la composition orale, loin de n'être qu'un texte en friche peut être considéré comme un possible acceptable du texte visé. Le recours à un logiciel de reconnaissance vocale ouvre ainsi des potentialités nouvelles dans les pratiques de scripteurs aguerris capables d'adapter leur niveau d'exigence vis-à-vis de leur texte à la finalité de la tâche d'écriture.

The development of efficient speech recognition software allows to write by speaking and leads to reconsider the borders of written and oral. This article questions the contribution of such a tool as part of a master course in professional writing, for a specific writing task, the summary. Forced into an oral composition without retouch, students achieved a scriptural act fully in the linearity of the oral and limiting the recourse to the spatializing of the written. The evaluation by students of their own productions and those of their peers, evaluation based on the concept of clarity, leads to conclude that, for the summary that relies on an explicit pre-text, the oral composition has not jeopardized the clarity of the text product: the "déjà-là" of the text in the oral composition, far from being a rough draft can be regarded as an acceptable target text. The use of speech recognition software offers new potential to professional writers who can adapt their level of requirement towards their text to the purpose of the writing task.

\section{INDEX}

Mots-clés : écriture de haut niveau, didactique de l'écriture, résumé, logiciel de reconnaissance vocale, clarté

Keywords : high-level writing, didactics of writing, summary, speech recognition software, clarity

\section{AUTEURS}

\section{MARIE-EMMANUELLE PEREIRA}

Aix-Marseille Université, Centre national de la recherche scientifique, École des hautes études en sciences sociales, CREDO, UMR 7308, F-13003, FranceESPÉ Aix-Marseille

\section{CHRISTINA ROMAIN}

Aix-Marseille Université, Centre national de la recherche scientifique, École des hautes études en sciences sociales, CREDO, UMR 7308, F-13003, FranceESPÉ Aix-Marseille

\section{VÉRONIQUE REY}

Aix-Marseille Université, Centre national de la recherche scientifique, École des hautes études en sciences sociales, CREDO, UMR 7308, F-13003, FranceESPÉ Aix-Marseille 